\title{
Rapid onset of altered mental status with progressive autonomic instability and hypoventilation in a 2-year-old male
}

\author{
Ramin Nazari ${ }^{1}$, Carlos A. Carmona Jr. ${ }^{2}$ \\ ${ }^{1}$ Department Pediatric Critical Care, ${ }^{2}$ Department of Pediatrics, Florida Hospital for Children, University of Central \\ Florida College of Medicine, Orlando, Florida-USA.E-mail: ccarmona08071984@gmail.com \\ Received: 12th January 2018, Revised: 6th April 2018, Accepted: 19th April 2018
}

SUMMARY: Nazari R, Carmona Jr CA. Rapid onset of altered mental status with progressive autonomic instability and hypoventilation in a 2-year-old male. Turk J Pediatr 2019; 61: 949-952.

\begin{abstract}
Anti N-methyl-D-aspartate receptor (NMDAR) encephalitis should be suspected in children who report with acute behavioral change, seizures, and non-specific movement disorders. While historically associated with adolescent females with ovarian teratoma, anti-NMDAR encephalitis has now been described in the pediatric population of both sexes. These patients display the neurological and psychiatric manifestations similar to females but lack a tumor. We report a two-year-old previously healthy young male who presented with alteration in speech, seizures, and extra pyramidal movement disorders. He was diagnosed with anti-NMDAR encephalitis with positive cerebrospinal fluid (CSF) serology for anti-NMDAR antibodies. In this case report, we describe the clinical presentation, course of illness, investigation, and management of our patient. We conclude by emphasizing three observations and insights from our case compared to what is mentioned in the literature.
\end{abstract}

Key words: tumor, CSF serology, pediatric neurology, NMDA receptors, movement disorders.

Anti-N-methyl-D-aspartate receptor (NMDAR) encephalitis was described initially by Dalmau et al. in 2007. It was originally characterized in young women with ovarian teratoma. However, there is an increasing number of cases reported in the male pediatric population with no concomitant findings of tumor. ${ }^{1}$

Anti-NMDAR encephalitis was defined in a series of 100 patients as a disorder that associates with antibodies against the NR1 subunit of the NMDA receptor. ${ }^{2}$ Most patients exhibit a unique constellation of symptoms including psychiatric manifestations, disturbance of consciousness, seizures, alterations of speech, movement disorders, autonomic instability, and central hypoventilation. ${ }^{1,2}$ The clinical diagnosis is suggested by the multistage clinical course and confirmed by the finding of NMDAR antibodies in the serum and cerebrospinal fluid. The mainstay of treatment includes tumor removal if present, immune-modulating therapies, and other medications for symptoms and supportive care. ${ }^{3}$

\section{Case Report}

After obtaining written informed consent, we wanted to report a previously healthy two-yearold male who initially displayed a change in mental status with aggressive behavior, crying, and episodes of "seeing things". He was said to have intermittent episodes of generalized shaking movements of his extremities with his eyes rolling back bilaterally. The patient had been admitted for a first-time seizure previously and was started on levetiracetam. During that admission, his neurologist noted epileptiform charges on electroencephalography (EEG) and made the diagnosis of complex partial 
seizures. He presented this time for unusual seizure-like activity such as lip smacking, jaw clenching, and abnormal gait. Over the next week, he became even more encephalopathic and agitated. He began having unusual body writhing movements, an inability to perform purposeful movements, and loss of speech. There was no purposeful response to verbal or tactile stimuli.

Pertinent labs showed a white blood cell (WBC) count of 23,200 cells/ $\mathrm{mm}^{3}$ (73 \% neutrophils, $19 \%$ lymphocytes). Creatine kinase (CK) level was 29,616 U/L (values for a normal male $>3$ months old: 39-3,08 $\mathrm{U} / \mathrm{L})$. The CK was elevated due to muscle involvement from the seizure-like activity and body writhing movements. Lumbar puncture (LP) studies came back days later revealing a cerebrospinal fluid (CSF) containing 24 leukocytes $/ \mathrm{mm}^{3}$ (including 36\% mononuclear cells), 291 red blood cells/ $\mathrm{mm}^{3}$, glucose of $54 \mathrm{mg} / \mathrm{dl}$, and CSF cultures negative for bacteria or suggestive of viral etiology. Serum glucose level was $107 \mathrm{mg} / \mathrm{dl}$. Urine toxicology screen was negative and thyroid studies were unremarkable. Initial brain magnetic resonance imaging (MRI) with contrast showed no abnormalities. Initial 24-hour period long term video electroencephalogram (EEG) was normal with no epileptiform discharges. Neck, chest, abdominal and pelvic computed tomography revealed no findings of mass lesions suggestive of neoplasm.

Due to the persistence of altered mental status and abnormal movements concerning infectious encephalitis, empiric antibiotics including vancomycin, cefotaxime, and acyclovir were initiated on day of admission. Lorazepam and diphenhydramine were included as needed for patient agitation. These treatments failed to alleviate the patient's altered mental status. Intravenous (IV) methylprednisolone (30 mg/kg/dose) was implemented on day three as concerns by the neurology team arose for acute disseminated encephalomyelitis (ADEM). They felt it was appropriate as it was too early to detect confirming lesions on MRI. Antibiotics and antivirals were discontinued when all culture results returned negative. Daily administration of IV methylprednisolone was discontinued after one week after the patient failed to improve. Intravenous Immunoglobulin (IVIG) $0.4 \mathrm{~g} / \mathrm{kg} /$ day was introduced for the following five days with minimal clinical change. $\mathrm{He}$ continued to exhibit continuous writhing movements and agitation thus needing clonazepam and haloperidol.

Our patient was transferred to the intensive care unit near day fourteen as he started developing autonomic instability and subsequent progression to hypoventilation and apnea. He was intubated and placed on mechanical ventilation. Clinical progression of our patient was suggestive of antiNMDAR encephalitis, but it was confirmed when serum antibodies to NMDAR were detected. Plasma exchange was subsequently introduced (for five days). A second cycle of IV methylprednisolone at the previous dose was also commenced. Sedation with fentanyl, midazolam, and dexmedetomidine were necessary for the persistent and increased agitation and movements. One dose of rituximab $\left(500 \mathrm{mg} / \mathrm{m}^{2}\right)$ was administered on day thirty of admission. He only had one treatment due to depletion of CD19 levels to a zero upon post treatment evaluation. After implementation of these therapies, his involuntary movements decreased. Repeat brain MRI showed non-specific white matter changes and interval development of atrophic changes. A repeat EEG only showed abnormal presence of diffuse theta activity.

A tracheostomy tube was placed to assist with his continued respiratory insufficiency; however, he was eventually weaned to room air. A gastrostomy tube was inserted for nutrition and hydration since adjustment of the patient's sedative and neuromodulator drugs rendered him slightly sedated and at risk for aspiration. He was eventually transferred to inpatient rehabilitation after being seizure free for some time and being back at his baseline per family. The patient continued showing gradual improvement after discharge.

\section{Discussion}

Anti-NMDA receptor encephalitis is a neuro-autoimmune disease arising from the generation of antibodies targeting synaptic proteins particularly the NR 1 subunit of the 
NMDA receptor. ${ }^{4}$ It was initially classified as a para neoplastic syndrome due to the strong association $(60 \%)$ with a teratoma or another tumor typ., ${ }^{4,5}$ Growing clinical experience suggests that this disorder may be better classified as a neuro-autoimmune syndrome in which antibodies form in response to several possible stimuli (i.e., tumor, infection) and cross react with synaptic proteins most commonly the $\mathrm{N}$-methyl-D-aspartate receptor (NMDAR). ${ }^{3}$ This direct antagonism of the receptor by antibodies mimics that of the pharmacological blockers of the receptor like ketamine. ${ }^{6}$

Usually the disease evolves in stages, but the clinical phases can vary in sequence, presentation, and severity. In $70 \%$ of patients, a prodromal phase lasts five to fourteen days and can include fever, malaise, inability to concentrate, nausea, diarrhea, vomiting, and headache. The psychotic or seizure phase follows with emotional and behavioral disturbances such as apathy and psychosis. Individuals may have seizures most commonly of the generalized tonic-clonic variety which are refractory to standard medical management. ${ }^{7,8}$ During the unresponsive phase, patients no longer follow verbal commands and appear mute and akinetic. The hyperkinetic phase is typified by autonomic instability such as cardiac arrhythmia, hypotension, hypertension, hypoventilation, and hyperor hypothermia. It may also present as lipsmacking, sustained jaw movements, and grimacing. ${ }^{7,8}$ The recovery phase is typically slow and symptoms may relapse especially in those with undetected or recurrent tumors and even no associated tumors. ${ }^{2,7,8}$ Clinical recovery may take up to years with some improving from the frontotemporal brain atrophy. ${ }^{9}$

Diagnosis is based on clinical symptoms and supporting results from brain MRI, EEG, and CSF. Infectious encephalitis, other autoimmune etiologies, drug intoxication, and even Hashimoto's encephalopathy must be excluded as the cause. ${ }^{7-9}$ Final diagnosis requires detection of anti-NMDA receptor antibodies in the patients' serum or $\mathrm{CSF}^{2,6}$ which should then be followed by imaging to detect an underlying teratoma or tumor. ${ }^{8,9}$
The management can be very effective with identification and treatment of a tumor if present. ${ }^{4}$ In patients without a tumor, firstline immunotherapy such as corticosteroids, intravenous immunoglobulin (IVIG), and plasma exchange might not be sufficient or effective as evidenced by our patient's response. A second-line immunotherapy (e.g., rituximab) is usually needed..$^{9,10}$ With addition of a secondline agent, such as cyclophosphamide, it still takes a significant amount of time to observe clinical improvement. While administrating immunomodulating agents, adverse effects of different agents such as severe neurologic deficits, gonadal toxicity, myelosuppression, malignancy, or death must be considered. $3,9,10$ In our patient, hypogammaglobulinemia was observed as a complication of rituximab deterring further treatment.

From our article, there are three main points to convey. First is that caregivers must strongly consider anti-NMDAR encephalitis early on in young pediatric patients having unexplained seizures and behavioral changes. Despite the median age of twenty-three with a range of five to seventy-six years old ${ }^{2}$ autoimmune etiologies such as anti-NMADR should be in the differential. ${ }^{11}$ This leads to quicker administration of immunotherapy with IVIG and methylprednisolone before obtaining CSF results. Even though our patient did not markedly progress with therapy initially, a case report from Matoq et al. ${ }^{12}$ describes significant early recuperation in two infants receiving early IVIG and methylprednisolone $+/$ - rituximab. We therefore suggest that the benefits of empiric therapy halting further evolution of the disease and minimizing morbidity far outweigh the risks. Second, we did not implement rituximab until after day thirty and after a five-day course of plasma exchange. This contrasts to Matoq's report where it was given as maintenance therapy prior to getting CSF findings. ${ }^{12} \mathrm{We}$ hope this encourages discussion about using rituximab or cyclophosphamide earlier before confirmatory CSF results if a patient can tolerate it. Additionally, this may prove better since the immunomodulators already delay to take effect. Third, autonomic instability is severe in infants and can leave a patient moribund. Caregivers must be prepared 
even though this stage is more common in adults and only occasional in children. ${ }^{12}$ This was evidenced by our patient needing to be intubated and mechanically ventilated for weeks before needing tracheostomy and gastrostomy tubes.

\section{REFERENCES}

1. Florance-Ryan N, Dalmau J. Update on anti-Nmethyl-D-aspartate receptor encephalitis in children and adolescents. Curr Opin Pediatr 2010; 22: 739744.

2. Dalmau J, Gleichman AJ, Hughes EG, et al. AntiNMDA-receptor encephalitis: case series and analysis of the effects of antibodies. Lancet Neurol 2008; 7: 1091-1098.

3. Kashyape P, Taylor E, Ng J, Krishnakuma D, Kirkham F, Whitney A. Successful treatment of two paediatric cases of anti-NMDA receptor encephalitis with cyclophosphamide: the need for early aggressive immunotherapy in tumour negative paediatric patients. Eur J Paediatr Neurol 2012; 16: 74-78.

4. Mirza MK, Pogoriler J, Paral K, et al. Adjunct therapeutic plasma exchange for anti-N-methylD-aspartate receptor antibody encephalitis: a case report and review of literature. J Clin Apher 2011; 26: 362-365.

5. Dalmau J, Tüzün E, Wu HY, et al. Paraneoplastic anti-N-methyl-D-aspartate receptor encephalitis associated with ovarian teratoma. Ann Neurol 2007; 61: 25-36.
6. Hughes EG, Peng X, Gleichman AJ, et al. Cellular and synaptic mechanisms of anti-NMDA receptor encephalitis. J Neurosci 2010; 30: 5866-5875.

7. Perry HE, Day GS, Dunn S et al. Anti-NMDA receptor encephalitis. The disorder, the diagnosis and the immunobiology. Autoimmun Rev 2012; 11: 863-872.

8. Iizuka T, Sakai F, Ide T, et al. Anti-NMDA receptor encephalitis in Japan: long-term outcome without tumor removal. Neurology 2008; 70: 504-511.

9. Wandinger KP, Saschenbrecker S, Stoecker W, Dalmau J. Anti-NMDA-receptor encephalitis: a severe, multistage, treatable disorder presenting with psychosis. J Neuroimmunol 2011; 231: 86-91.

10. Dalmau J, Lancaster E, Martinez-Hernandez E, Rosenfeld MR, Balice-Gordon R. Clinical experience and laboratory investigations in patients with antiNMDAR encephalitis. Lancet Neurol 2011; 10: 6374.

11. Gable MS, Gavali S, Radner A, et al. Anti-NMDA receptor encephalitis: report of ten cases and comparison with viral encephalitis. Eur J Clin Microbiol Infect Dis 2009; 28: 1421-1429.

12. Matoq AA, Rappoport AS, Yang Y, O’Babatunde J, Bakerywala R, Sheth RD. Anti-NMDA-receptor antibody encephalitis in infants. Epilepsy Behav Case Rep 2015; 4: 99-101. 